\title{
OST1 phosphorylates ICE1 to enhance plant cold tolerance
}

\author{
LANG ZhaoBo $^{1 *} \&$ ZHU JianKang ${ }^{1,2}$ \\ ${ }^{1}$ Shanghai Center for Plant Stress Biology, Shanghai Institutes for Biological Sciences, Chinese Academy of Sciences, Shanghai 200032, \\ China; \\ ${ }^{2}$ Department of Horticulture and Landscape Architecture, Purdue University, West Lafayette, Indiana 47907, USA
}

Received February 11, 2015; accepted February 12, 2015; published online February 13, 2015

Citation: $\quad$ Lang ZB, Zhu JK. OST1 phosphorylates ICE1 to enhance plant cold tolerance. Sci China Life Sci, 2015, 58: 317-318, doi: 10.1007/s11427-015-4822-7

Low temperature is one of the most important environmental stresses that affect plant survival, growth and development. In response to cold stress, hundreds of genes are upor down-regulated in plants [1]. The C-repeat binding factor $(\mathrm{CBF})$ regulon is the best-understood cold-responsive transcriptional module [1]. In Arabidopsis, there are three $C B F$ genes, i.e., $C B F 1, C B F 2$ and $C B F 3$. They are rapidly induced when plants are exposed to low temperature. The $C B F$ genes encode transcription factors that activate the expression of many downstream cold responsive (COR) genes, which in turn encode proteins that protect plant cells from freezing damage.

Multiple transcription factors act as upstream regulators of $C B F$ expression in response to cold stress. MYB15 and EIN3 (ethylene insensitive 3) are negative regulators of $C B F \mathrm{~s}$, whereas ICE1 (inducer of CBF expression1) and CAMTA (calmodulin binding transcription activator) proteins are positive regulators. In addition to these upstream transcription factors that directly bind to $C B F$ promoters, two enzymes, HOS1 (high expression of osmotically responsive gene1) and SIZ1 (SAP and Miz 1), regulate $C B F$ expression indirectly by changing ICE1 protein stability and activity $[2,3]$. HOS1 is an ubiquitin E3 ligase involved in the ubiquitination and proteosomal degradation of ICE1 under cold stress [2]. In contrast, SIZ1 is a SUMO (small ubiquitin related modifier) E3 ligase involved in the stabilization of ICE1 protein [3]. Besides sumoylation and ubiquitination, protein phosphorylation may also play an im-

*Corresponding author (email: lang.katrina@gmail.com) portant role in the regulation of ICE1 activity under cold stress [4]. However, the protein kinase involved in ICE1 phosphorylation in response to cold stress has been a mystery.

Recently, the research group led by Yang ShuHua at China Agricultural University discovered that the protein kinase OST1 (open stomata 1) phosphorylates ICE1 under cold stress to stabilize and activate ICE1 and thus facilitates $C B F$ expression (Figure 1). Their study, for the first time, revealed a protein kinase that is critical for freezing tolerance in plants by directly phosphorylating ICE1. Ding et al. [5] identified OST1 through a reverse genetics screen in Arabidopsis using a freezing tolerance assay. They found that dysfunction of OSTI in ost 1 mutants compromised the freezing tolerance of Arabidopsis plants. The ost 1 mutations also impaired the cold induction of $C B F$ expression, suggesting that OST1-mediated cold tolerance is CBFdependent. The conclusion was further supported by the enhanced freezing tolerance and increased cold-induced expression of $C B F S$ in $O S T 1$-overexpression plants.

Ding et al. found that OST1 interacted with ICE1 both in vitro (using yeast two hybrid and protein pull down assays) and in vivo (using co-immunoprecipitation and bimolecular fluorescence complementation assays). ICE1 could be phosphorylated by OST1. They showed that the kinase activity of OST1 was rapidly activated by cold stress. Serine-278 of ICE1 was identified as a major site of phosphorylation by OST1. The defect in freezing tolerance of icel mutant plants could be rescued by wild type ICEI but not mutated form of ICE1 (ICE1 $\left.1^{S 278 A}\right)$. Compared to unphos- 


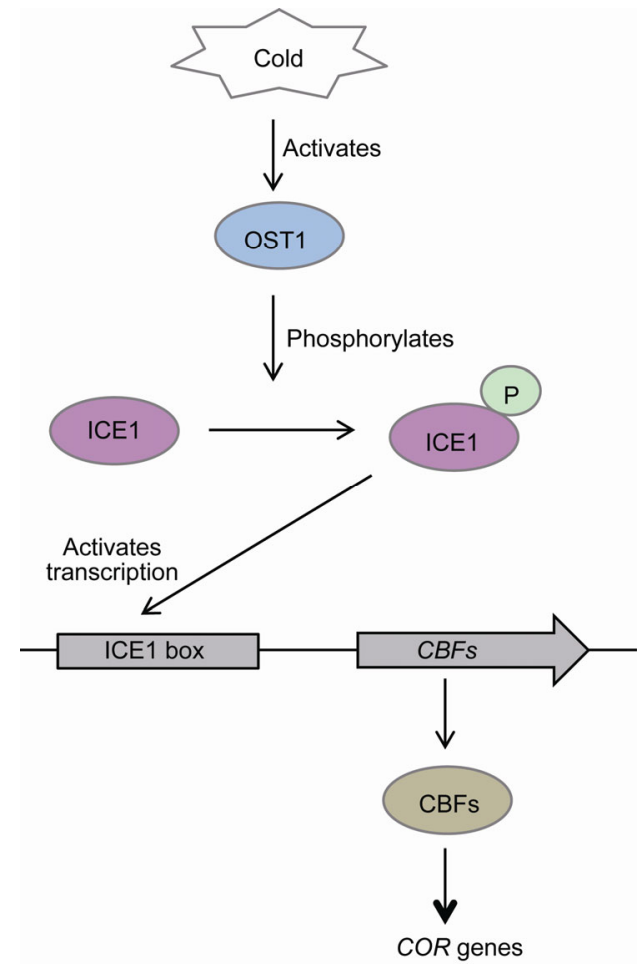

Figure 1 (color online) Model showing that cold-activated OST1 phosphorylates and stabilizes ICE1, which in turn binds to the cis-elements on $C B F$ gene promoters and activates the expression of $C B F s$ and downstream cold responsive (COR) genes, leading to enhanced freezing tolerance.

phorylated ICE1, the OST1-phosphorylated ICE1 has an enhanced binding affinity to the $C B F 3$ promoter in vitro. OST1 was shown to facilitate ICE1 transcriptional activity using a transient transactivation assay with the proCBF3:: GUS as a reporter. These results suggested that phosphorylation of ICE1 at S278 by OST1 is critical for ICE1 function in plant cold responses.

The authors also found that OST1 functions in stabilizing ICE1 protein under cold conditions. Overexpression of OST1 inhibited cold-induced degradation of ICE1, and dysfunction of OST1 in ost1 mutant plants caused faster ICE1 degradation in response to cold. Overexpression of OSTI was also shown to reduce cold-induced ubiquitination of ICE1, which was mediated by HOS1. ICE1 ${ }^{\text {S278D }}$ that mimics phosphorylation at S278 failed to bind to HOS1. All these results suggest that OST1-mediated phosphorylation of ICE1 enhances ICE1 stability by reducing ubiquitination of
ICE1 under cold conditions. Interestingly, Ding et al. found that OST1 can physically interact with HOS1, and can interfere with the interaction between HOS1 and ICE1. Therefore, it appears that OST1 enhances ICE1 stability by both phosphorylation and physically competing with binding to HOS1. It would be interesting to test whether the kinase-dead mutant form of OST1 can still enhance ICE1 stability in plants.

OST1 is also known as SnRK2.6 (SNF1-related kinase 2.6), which is a well-known kinase in ABA signaling mainly in guard cells. Under water stress conditions, ABA accumulates and binds to receptors causing their conformational change. The activated ABA receptors then interact with and inhibit PP2C protein phosphatases, thus relieving OST1 from inhibition by the PP2Cs. How OST1 is activated by ABA is well understood. Is ABA involved in cold stress activation of OST1? Ding et al. showed that deficiency in ABA production in aba2-21 mutant plants did not compromise cold-induced activation of OST1. In addition, OST1 was activated after $0.5-1.5 \mathrm{~h}$ of cold treatment, which occurred before any cold stress induced ABA accumulation. These results indicated that ABA plays little if any role in cold-induced activation of OST1. It would be exciting to understand how OST1 is activated by cold stress.

In summary, Ding et al. reported the first protein kinase involved in cold signaling that directly phosphorylates ICE1. Their work makes an important contribution to our understanding of cold stress signaling and cold tolerance in plants.

1 Zhu J, Dong CH, Zhu JK. Interplay between cold-responsive gene regulation, metabolism and RNA processing during plant cold acclimation. Curr Opin Plant Biol, 2007, 10: 290-295

2 Dong $\mathrm{CH}$, Agarwal M, Zhang Y, Xie Q and Zhu JK. The negative regulator of plant cold responses, HOS1, is a RING E3 ligase that mediates the ubiquitination and degradation of ICE1. Proc Natl Acad Sci USA, 2006, 103: 8281-8286

3 Miura K, Jin JB, Lee J, Yoo CY, Stirm V, Miura T, Ashworth EN, Bressan RA, Yun DJ, Hasegawa PM. SIZ1-mediated sumoylation of ICE1 controls CBF3/DREB1A expression and freezing tolerance in Arabidopsis. Plant Cell, 2007, 19: 1403-1414

4 Chinnusamy V, Zhu J and Zhu JK. Cold stress regulation of gene expression in plants. Trends Plant Sci, 2007, 12: 444-451

5 Ding Y, Li H, Zhang X, Xie Q, Gong Z, Yang S. OST1 kinase modulates freezing tolerance by enhancing ICE1 stability in Arabidopsis. Dev Cell, 2015, 32: 278-289

Open Access This article is distributed under the terms of the Creative Commons Attribution License which permits any use, distribution, and reproduction in any medium, provided the original author(s) and source are credited. 